\title{
Molecular Profiling of Neprilysin Expression and its Interactions with SARS-CoV-2 Spike Proteins to Develop Evidence Base Pharmacological Approaches for Therapeutic Intervention
}

\author{
Arun HS Kumar
}

\begin{abstract}
Introduction: Neprilysin due to its peptidase activity is involved in several physiological and pathological processes. Recently our group has reported the association of neprilysin with angiotensin-converting enzyme 2 (ACE2) network proteins which facilitate the entry of SARS-CoV-2 virus. The potential role of neprilysin beyond its peptidase activity is not known. Materials and Methods: Using the established sequence analysis and molecular docking tools, this study evaluated the molecular profile of neprilysin interaction with SARSCoV-2 virus proteins. Results: Human neprilysin protein showed a significant sequence similarity with SARS-CoV-2 spike protein, which was further confirmed by observation of considerable interaction in the molecular docking. Human neprilysin protein was also found to additionally interact with SARS-CoV-2 proteins facilitating virus replication. The potential of neprilysin inhibitors (Sacubitril and Sacubitrilat) to interfere with neprilysin and SARS-CoV-2 proteins interactions was assessed. The neprilysin inhibitors showed binding efficacy within therapeutically feasible concentration range (1 to $150 \mathrm{uM}$ ). Conclusion: This study while reporting a novel role of neprilysin as potential receptor for SARS-CoV-2 virus, highlights the merit in assessing clinical efficacy of neprilysin inhibitors for the management of SARS-CoV-2 infection.
\end{abstract}

Key words: Neprilysin, SARS-CoV-2, Receptor pharmacology, Sacubitril, Sacubitrilat, Virus entry.

\section{INTRODUCTION}

Neprilysin is a widely expressed peptidase located on the cell surface and has very diverse substrate specificity. ${ }^{1-3}$ Due to its diverse substrate specificity it is involved in regulating variety of physiological (electrolyte balance, blood pressure regulation, analgesia) and pathological ( $\mathrm{T}$ cell lymphoma, Alzheimer's, diabetes, diarrhoea) process. The biochemical function of neprilysin is to hydrolyse peptides by cleaving them at their $\mathrm{N}$-terminal side of hydrophobic amino acid residues. ${ }^{1-6}$ The peptidase activity of the neprilysin is seen as its major physiological role which is achieved by modulating signalling of a variety of peptides in various organ systems (Mangiafico et al., 2013). Neprilysin is also reported to cleave several mitogenic peptides and hence can curtail development of tumours., ${ }^{3,5}$ The major expression of neprilysin in mammals is reported in kidneys, lungs, GI tract and neuronal cells. ${ }^{3,5,7,8}$ Neprilysin is also located on the neutrophils and in lung, wherein respiratory irritants are reported to downregulate its expression and trigger inflammatory response. ${ }^{9,10}$ In contrast plasma neprilysin activity was reported to be increased in preclinical models associated with systemic inflammation, such as

Cite this article : Kumar AHS. Molecular Profiling of Neprilysin Expression and its Interactions with SARS-CoV-2 Spike Proteins to Develop Evidence Base Pharmacological Approaches for Therapeutic Intervention. BEMS Reports. 2021;7(2):15-20. obesity and insulin resistance. ${ }^{1,2,9,10}$ Neprilysin is also expressed on common lymphoid progenitors, which give rise to cells ( $\mathrm{T}, \mathrm{B}$ and NK cells) having significant role in tissue inflammation and immune response..$^{6-8,11}$ However it is not clear if changes in the activity of neprilysin is a cause or consequence of inflammation. Neprilysin inhibitors are approved for clinical use in human patients with heart failure. ${ }^{4,12,13}$ In the past neprilysin inhibitors have shown efficacy as therapeutics for several diseases associated with systemic inflammation. ${ }^{1,414}$ Although the role of neprilysin with airway inflammatory cascade is previously reported. ${ }^{1,4,12,14}$ its role in inflammatory cascade triggered by viruses in human patients is not clear. Our group recently reported the interaction of SARS-CoV-2 virus with several angiotensinconverting enzyme 2 (ACE2) network proteins (DPP4 and Meprin A alpha) with superior efficacy and neprilysin was observed as one of the ACE2 network proteins in this study. ${ }^{15}$ A low lymphocyte count among patients with SARS-CoV-2 infection is reported to be significantly associated with fatal outcome. Considering the expression of neprilysin on lymphocytes and its role in regulation of peptides

\section{Arun HS Kumar}

School of Veterinary Medicine, University College Dublin, Belfield, Dublin, IRELAND.

\section{Correspondence}

Dr. Arun HS Kumar, DVM,

(Pharmacology), School of Veterinary Medicine, University College Dublin, Belfield, Dublin-04, IRELAND.

Phone no: 0035317166230 E-mail: arun.kumar@ucd.ie

\section{History}

- Submission Date: 10-04-2021

- Review completed: 20-04-2021;

- Accepted Date: 24-04-2021.

DOI : 10.5530/bems.7.2.7

Article Available online http://www.bemsreports.org

Copyright

(C) 2021 Phcog.Net. This is an openaccess article distributed under the terms of the Creative Commons Attribution 4.0 International license. 
interacting with ACE2, this study tested the hypothesis that SARS-CoV-2 virus can directly interact with neprilysin. Hence molecular interactions of neprilysin and its inhibitors with SARS-CoV-2 proteins were assessed to establish their potential therapeutic merit.

\section{MATERIALS AND METHODS}

Protein network and sequence analysis: The neprilysin protein network was analysed using the STRING database (https://string-db.org/cgi/info. pl). ${ }^{16}$ The STRING database was searched using the neprilysin as protein name and Homo sapiens as organism. The Basic Local Alignment Search Tool (BLAST) was used to identify the regions of similarity between sequences of the neprilysin network proteins identified in the string database. Subsequent to initial alignment with all network proteins, similarities were further assessed between neprilysin, DPP4 and ACE2. To know the potential interaction of neprilysin with SARS-CoV-2 spike proteins the similarity between their sequences was compared. ${ }^{15-17}$ Neprilysin expression analysis: Expression of neprilysin in various human tissues was analysed by comparing the gene, RNA and protein levels reported in following databases. ${ }^{17,18}$ (https://genevisible.com/ tissues/HS/Gene\%20Symbol/MME, http://biogps.org/\#goto=welcome, and https:/www.proteinatlas.org/ENSG00000196549-MME). Tissue specific protein expression was accessed on 14 ${ }^{\text {th }}$ August 2020 (https:// www.proteinatlas.org/ENSG00000196549-MME/tissue).

SARS-CoV-2 surface proteins: The reported SARS-CoV-2 target proteins were searched in the protein data bank (https://www.rcsb. org/) and uniport database (https://www.uniprot.org/peptidesearch/) as reported previously. ${ }^{15,19}$ The following SARS-CoV-2 proteins were identified for binding analysis:

\begin{tabular}{cc}
\hline PDB/Protein ID & Brief Description \\
\hline 6 W6Y & ADP ribose phosphatase of NSP3 \\
6LXT & Post fusion core of S2 subunit \\
6Y2E & SARS-CoV-2 main protease \\
7JWB & SARS-CoV-2 Spike ectodomain \\
6 ZB5 & SARS-CoV-2 Spike protein open confirmation \\
6 ZB4 & SARS-CoV-2 Spike protein close confirmation \\
7DDN & SARS-CoV-2 S protein at open state \\
7DDD & SARS-CoV-2 S protein at closed state \\
7AD1 & SARS-CoV-2 Spike \\
7DK3 & SARS-CoV-2 S trimer, S-open \\
7A93 & SARS-CoV-2 Spike Glycoprotein with 2 RBDs Erect \\
7KDI & SARS-CoV-2 Spike furin cleaved
\end{tabular}

Protein 3D structure and molecular docking: The 3D structure of SARS-CoV-2 targets listed above were processed for molecular docking as described previously. ${ }^{15,19-21}$ The structures of neprilysin inhibitors (Sacubitril and Sacubitrilat) were accessed from PubChem database and were processed for molecular docking as described previously using the Chimera software and AutoDock Vina (version 1.5.4) ) $^{19,20,22}$

Simulation of dose response curves: Dose-response curves were modelled based on nonlinear regression analysis as reported before. ${ }^{19,23}$

Interaction analysis using Ligplot: The protein-protein or proteinligand interactions was evaluated using the LigPlot software. The PDB output files from molecular docking were inputted into the LigPlot and the intermolecular interactions and their features (hydrogen bonds, hydrophobic contacts, and atom accessibilities) were assessed. Hydrogen bonds are represented by dashed lines. The amino acid residues of the protein involved in the molecular interactions are represented by an arc with spokes emerging towards the ligand atoms in contact. ${ }^{24,25}$

\section{RESULTS}

The network analysis of human neprilysin protein in the string database showed 10 proteins in its primary network (Figure 1A). The Basic Local Alignment Search Tool (BLAST) analysis of the sequence of the neprilysin network proteins, showed a very weak identify $(0.082 \%)$ (Table 1, Figure 1B). We and others have reported the role of DPP4 and ACE2 proteins as cell surface receptors for entry of coronaviruses. ${ }^{15,26}$ As both DPP4 and ACE2 were observed in the neprilysin protein work, BLAST was use to assess the similarities between these three proteins. Compared to the general neprilysin network proteins, both DPP4 and ACE2 showed significantly better identity $(2.205 \%, 112$ similar positions) in their sequence (Table 1, Figure 1B). Which suggested neprilysin may be a potential receptor for coronaviruses. Hence the SARS-CoV-2 Post fusion core of S2 subunit (PDB ID: 6LXT), which is reported to be an important component of spike protein interacting with ACE2 receptor was compared to neprilysin. The sequence similarities (235 similar positions) between neprilysin and 6LXT was significantly better (9.058 Vs 2.205\%) than that between neprilysin and DPP4/ACE2 (Table 1, Figure 1B).

The expression profile of receptors is essential to effectively correlate the associated pathology and as well for designing rationale therapeutic measures. Hence the relevant database were analysed to profile the gene, transcript and protein expression pattern of neprilysin in various human tissues (Figure 2). Differences between the relative expression of neprilysin RNA, gene and protein were observed across various human tissues/organs, which although not surprising was nevertheless interesting (Figure 2). Highest expression of neprilysin RNA was observed in adipocytes, whole blood, olfactory bulb and lymphoma (Figure 2A). In contrast highest expression of neprilysin gene was observed in glomerulus, jejunum and nephrons (Figure 2B). The RNA and gene expression pattern of neprilysin in human tissue differed from its protein expression pattern, although gene and protein expression pattern was similar (Figure 2B and C). Highest expression of neprilysin protein was observed in small intestines, followed by that in duodenum, colon, kidneys, and granulocytes (Figure 2C). Hence considering this expression pattern of neprilysin, it is likely that gastrointestinal, renal and immune physiology are predominantly influenced by factors interfering with neprilysin function. The symptoms of nausea, diarrhoea, generalised inflammation observed with SARS-CoV-2 infection does correlate with the expression pattern of neprilysin in human tissues. Hence to assess if SARS-CoV-2 virus proteins can interact with neprilysin, molecular docking of the selected combination of protein (Table 2) was performed. SARS-CoV-2 virus proteins (6LXT, 6Y2E, 6W6Y, 6ZB5) were observed to significantly interact with neprilysin through formation of hydrogen bonds in sufficient numbers for the interaction to be biochemically feasible (Figure 3A, Table 2). The interaction of neprilysin with 6LXT was observed at Glu646, His587, His583, Asp950 regions with 44 hydrogen

Table 1: Basic Local Alignment Search Tool (BLAST) analysis for sequence similarities.

$\begin{array}{cccc} & \begin{array}{c}\text { Identical } \\ \text { positions }\end{array} & \begin{array}{c}\text { Similar } \\ \text { positions }\end{array} & \text { Identity(\%) } \\ \begin{array}{c}\text { Neprilysin network } \\ \text { proteins }\end{array} & 1 & 2 & 0.082 \\ \text { Neprilysin, ACE2, DPP4 } & 25 & 112 & 2.205 \\ \text { Neprilysin and 6LXT } & 126 & 235 & 9.058\end{array}$


bonds, suggesting the possibility of neprilysin serving as a receptor for SARS-CoV-2 spike protein (Figure 3B, Table 2). Of considerable interest was the superior interaction (more number of hydrogen bonds) of neprilysin with SARS-CoV-2 proteins (6Y2E, 6W6Y) involved in its replication (Table 2).

Sacubitril and Sacubitrilat are neprilysin inhibitors, which are approved for clinical use in humans. Hence the binding affinity of these two drugs against SARS-CoV-2 proteins was assessed. The binding affinity (Figure 4A) and the $\mathrm{IC}_{50}$ (Table 3) of Sacubitril and Sacubitrilat against the SARS-CoV-2 proteins (6LXT, 6Y2E and 6W6Y) were observed to be within therapeutically feasible concentration (Figure 4A, Table 3). Based on the $\mathrm{IC}_{50}$ values, simulated dose response curves for Sacubitril and Sacubitrilat were generated for optimal estimation of therapeutic concentration range ( 1 to $150 \mathrm{uM}$ ) (Figure $4 \mathrm{~B})$. Sacubitril showed superior efficacy than sacubitrilat in interacting with SARS-CoV-2 targets (Figure 4B). Sacubitril was observed to form 26 hydrogen bonds with 6LXT (selectively at Asp950, Gln949 and Gln1180) suggesting

B

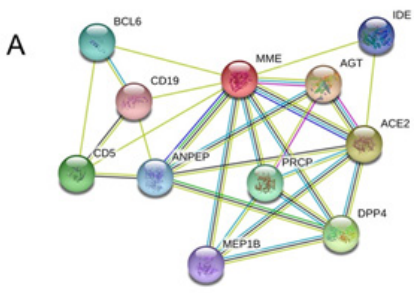

Alignment with Neprilysin network proteins

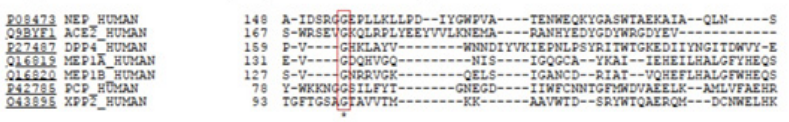

Alignment between ACE2, DPP4 and Neprilysin

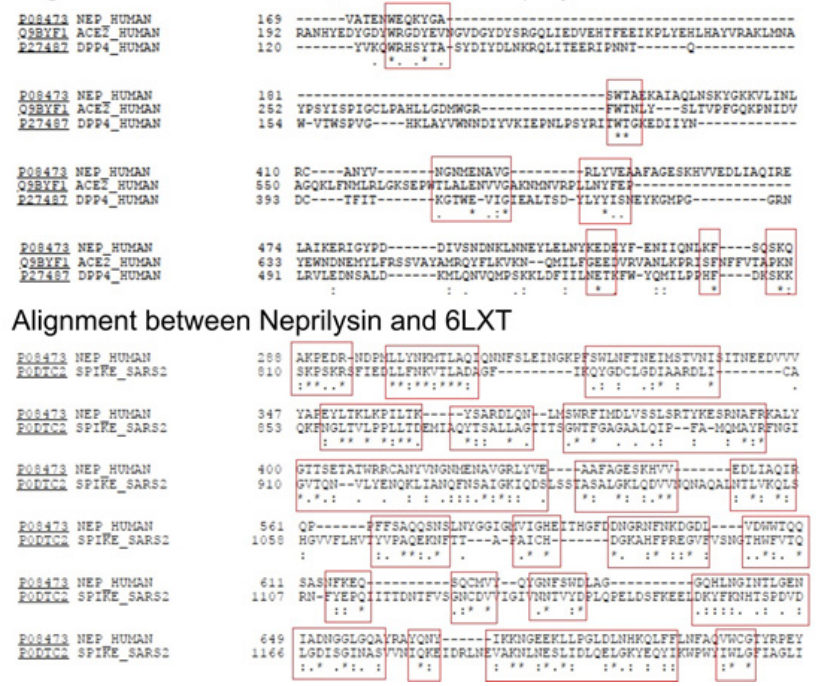

Figure 1: (A) Network protein analysis of human neprilysin protein (MME) in string database, showing 10 proteins in the primary network. The following proteins were identified: Insulin-degrading enzyme (IDE), Angiotensinconverting enzyme 2 (ACE2), Meprin A subunit beta (MEP1B), Lysosomal Pro-X carboxypeptidase (PRCP), Dipeptidyl peptidase 4 (DPP4), Angiotensinogen (AGT), Aminopeptidase N (ANPEP), T-cell surface glycoprotein (CD5), B-lymphocyte antigen (CD19), B-cell lymphoma 6 protein (BCL6). (B) The similarities in the sequence (red box) of human neprilysin and its network proteins and SARS-COV2 Post fusion core of S2 subunit (spike protein) are shown.<smiles>[C]1[CH]CC1</smiles>

significant binding affinity (Figure 4C, Table 2) and its potential to block interaction of neprilysin with the SARS-CoV-2 spike protein.

\section{DISCUSSION}

Several cell surface receptors are known to facilitate the entry of viruses, which can be target for therapeutic intervention. ${ }^{15,26}$ This study reports an unexpected observation from network protein analysis, which resulted in identification of neprilysin as a potential receptor for the key proteins of recently reported SARS-CoV-2 virus. The molecular interaction of neprilysin was observed with SARS-CoV-2 virus post fusion core of S2 subunit (6LXT), spike protein open confirmation (6ZB5), ADP ribose phosphatase of NSP3 (6W6Y) and its main protease (6Y2E) suggesting neprilysin may facilitate both viral attachment, entry and its replication.

Table 2: Molecular docking and Ligplot analysis.

$\begin{array}{cccc}\text { Protein } & \text { Ligand } & \text { Hydrophilic interaction } & \text { H-Bonds } \\ \text { Neprilysin } & \text { 6LXT } & \text { Glu646, His587, His583, Asp950 } & 44 \\ \text { Neprilysin } & \text { 6ZB5 } & \text { Didn’t evaluate } & 168 \\ \text { Neprilysin } & \text { 6Y2E } & \text { Didn’t evaluate } & 87 \\ \text { Neprilysin } & \text { 6W6Y } & \text { Didn’t evaluate } & 356 \\ \text { 6LXT } & \text { Sacubitril } & \text { Asp950, Gln949, Gln1180 } & 26 \\ \text { Neprilysin } & \text { Sacubitril } & \text { His587, His 583, Glu646, Ser517 } & 7\end{array}$

\section{Expression of neprilysin (MME) in humans tissues}

A
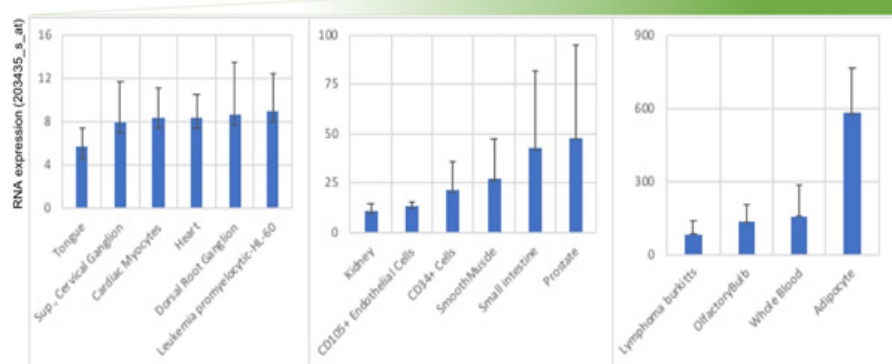

B
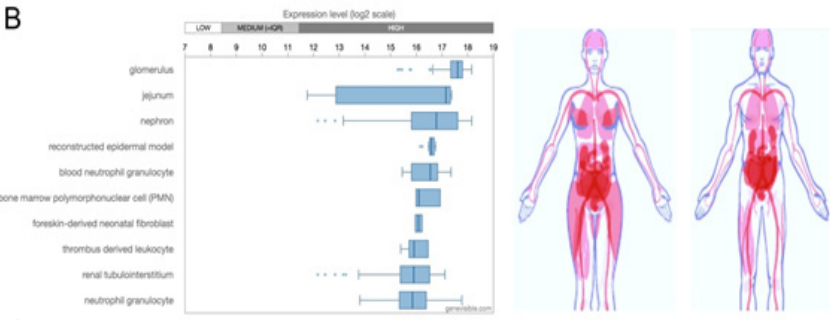

C

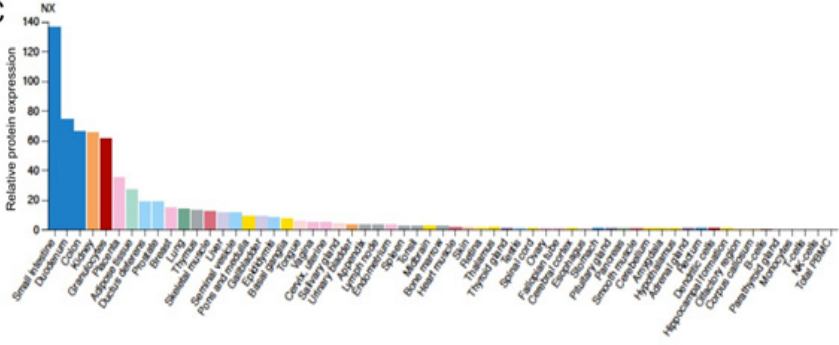

Figure 2: Expression profile of neprilysin RNA (A), gene (B) and protein (C) in various human tissues/organ. The green scale bar on the top indicates the degree of expression, with higher intensity of colour indicating higher expression. The image corresponding to section $B$, indicates the various organs (in females and males) where neprilysin gene is expressed. 
This novel role of neprilysin as a receptor for viruses is not reported before.

Viruses which spread at a pandemic scale are unlikely to depend on a single receptor type for it attachment and entry into the host cell. Dependency on multiple receptor types has been shown for many strains of coronaviruses reported previously. ${ }^{15,26}$ Most of these strains of coronaviruses have caused infections in large scale, both in humans and animals despite the endemic nature of the infections. ${ }^{27}$ SARS-CoV-2
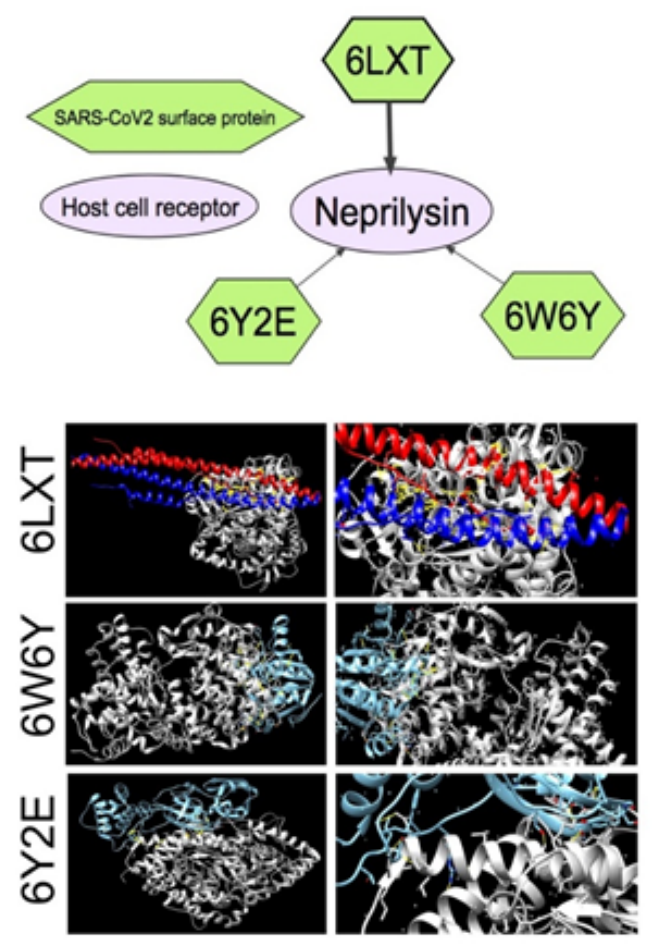

B
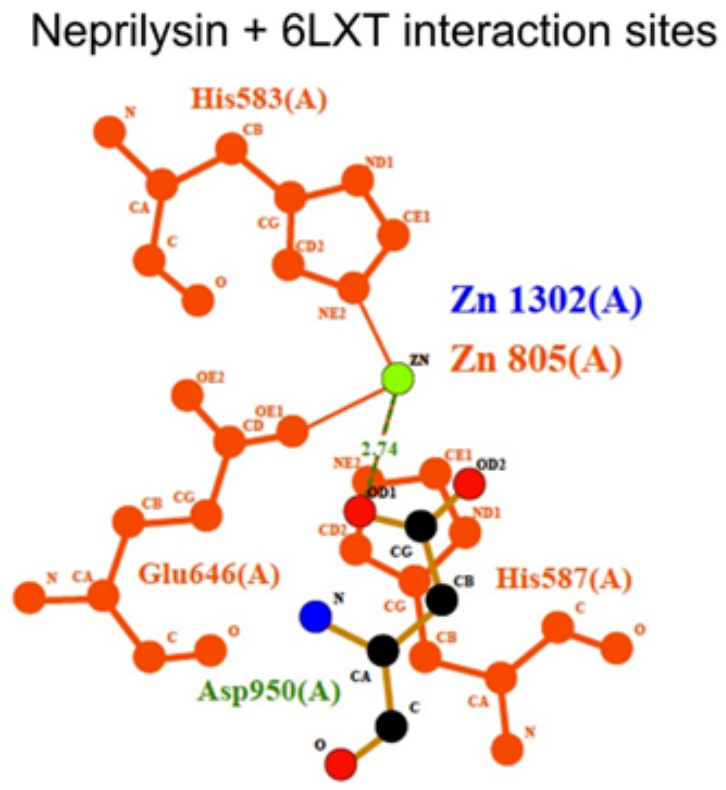

Figure 3: (A) Molecular docking of neprilysin with SARS-COV2 proteins (6LXT, $6 \mathrm{W6Y}, 6 \mathrm{Y} 2 \mathrm{E})$. Representative images of each of the interaction combinations with their respective magnified view (right) are shown. (B) Ligplot assessment of the molecular interacting sites between neprilysin and 6LXT is shown. is the recently reported strain of coronaviruses which has spread to a pandemic scale, ${ }^{15,26,27}$ and paraphs it does utilize multiple receptor types at least in non-experimental settings for attachment and entry into the host cells. We have recently reported the role of ACE2 network proteins in facilitating SARS-CoV-2 virus attachment and entry into the host cells, with some of the network proteins (DPP4, Meprin A and XPNPEP2) showing superior molecular interactions with SARS-CoV-2 virus spike proteins compared to ACE2 ${ }^{15}$ Coincidental observation of neprilysin

Table 3: Molecular docking.

\begin{tabular}{|ccccc|}
\hline $\mathrm{IC}_{50}$ (uM) & Neprilysin & $\begin{array}{c}6 \mathrm{6Y2E} \\
\text { (Protease) }\end{array}$ & $6 \mathrm{LXT}$ & $6 \mathrm{W6Y}$ \\
\hline Sacubitril & $9.33 \pm 0.56$ & $34.03 \pm 0.34$ & $0.91 \pm 0.01$ & $9.22 \pm 0.08$ \\
Sacubitrilat & $157.69 \pm 5.41$ & $91.98 \pm 3.71$ & $45.19 \pm 0.76$ & $133.17 \pm 7.47$
\end{tabular}
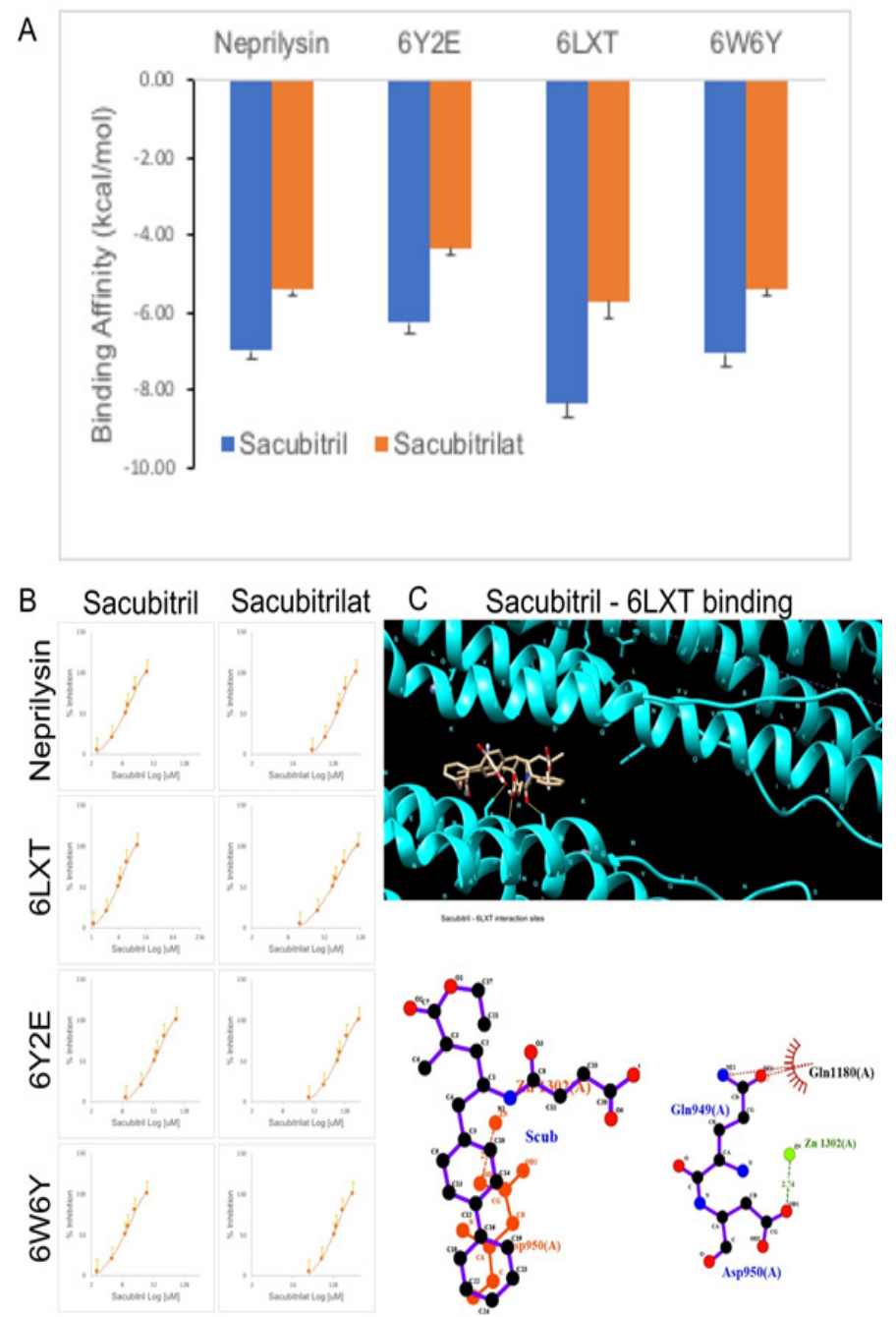

Figure 4: Molecular docking analysis of neprilysin inhibitors (Sacubitril and Sacubitrilat) with neprilysin and SARS-COV2 proteins (6LXT, 6W6Y, 6Y2E). (A) Binding affinity of neprilysin inhibitors with their targets is represented as bar graph. The data is presented as mean \pm SD of top nine interacting sites. (B) The simulated dose response curves of the neprilysin inhibitors with their targets is shown. The data is presented as mean \pm SD of three sigma deviations from the mean $\mathrm{IC}_{50}$ value. (C) Ligplot assessment of the molecular interacting sites between Sacubitril and 6LXT is shown. 
association with the ACE2 network protein and its sequence similarities with the SARS-CoV-2 spike protein observed in this study suggest the possibility of neprilysin being a receptor for coronaviruses similar to DPP4 and ACE2. ${ }^{15,27}$ Additionally superior molecular interaction of neprilysin with SARS-CoV-2 proteins (6W6Y, 6Y2E) regulating its replication was also observed. Although neprilysin is predominantly expressed on cell membrane, a few studies have reported its subcellular localisation, ${ }^{3,28}$ suggesting the potential role of neprilysin beyond its peptidase activity against natriuretic peptides. Facilitating attachment, entry and replication of virus in the host cell may be one alternative role of neprilysin, which merits further investigation.Loss of smell and taste is reported to an early symptom of SARS-CoV-2 infection. While the exact mechanisms responsible for loss of this physiology is not known, ${ }^{29}$ it is interesting to note that higher expression of neprilysin is observed in olfactory bulb. In contrast expression of neprilysin was least in the tongue. The differential loss of smell and taste sensation in SARS-CoV-2 infection are perhaps mediated by different pathways, with a potential role of neprilysin in regulating the sense of smell. The predominant expression of neprilysin in GI tract, kidneys and granulocytes, does correlate with the symptoms of nausea, diarrhoea, thrombosis and systemic inflammation observed in patients with SARS-CoV-2 infection. ${ }^{29}$ Respiratory irritants and pathogens (parainfluenza virus and rat coronavirus) are reported to interact with neprilysin on neutrophils and in lung epithelium to trigger inflammatory responses. ${ }^{2,9,10}$ These prior studies are in concurrence with this study reporting the potential of neprilysin as a receptor for SARS-CoV-2 virus. Besides these a soluble circulating form of neprilysin is also reported in several body fluids, ${ }^{30}$ which together with high expression of neprilysin in whole blood may substantiate the systemic nature of inflammation observed in SARS COV2 infection. Neprilysin expression was also highest in the adipose tissue, which paraphs support the incidence of higher mortality rate in obese patients with SARS-CoV-2 infection. ${ }^{3,8,11}$ With fibrinogen as its substrate, neprilysin can regulate fibrin formation by thrombin. ${ }^{31}$ Hence factors inhibiting neprilysin can enhance fibrinogen levels and lead to intravascular coagulation. The molecular interactions observed in this study does indicate the potential of SARS-CoV-2 spike proteins to interact and inhibit neprilysin activity, weather this is the potential mechanism of disseminated intravascular coagulation observed in SARSCoV-2 infections remains to be validated. Several studies have associated neprilysin activity with negative remodelling of pulmonary and vascular structures, including increased microvascular permeability. ${ }^{6,8,10,32,33}$ The correlation of these features with symptoms observed in SARS-CoV-2 infections together with the molecular interactions between SARS$\mathrm{CoV}-2$ proteins and neprilysin reported in this study does support the notion for neprilysin being a potential receptor for interaction with SARS-CoV-2 virus. Further the higher catalytic activity of neprilysin then $\mathrm{ACE} 2^{12,13}$ may be more favourable for the enveloped virus to enter host cells.

Neprilysin inhibitors (Sacubitril and Sacubitrilat) are currently approved for clinical use and have shown efficacy in the treatment of acute diarrhoea and heart failure. ${ }^{12,13}$ The binding efficacy of both sacubitril and sacubitrilat against SARS-CoV-2 proteins (6LXT, 6W6Y and 6Y2E) were within therapeutically feasible range, indicating their potential in not only preventing virus attachment and entry into host cell but also the potential to prevent virus replication. This ability of neprilysin inhibitors to target full cycle of virus entry and replication can lead to synergistic outcomes and improved efficacy. Further the synergistic efficacy of neprilysin inhibitors could be a consequence of targeting both neprilysin as well as SARS-CoV-2 proteins independently. This dual targeting of both host cell and virus proteins in addition to curtailing the pathogenesis of the virus can also be helpful to harness the collateral benefits from neprilysin inhibition. Recent studies have supported the benefits from neprilysin inhibition by reducing the pro-inflammatory cytokines and neutrophil count in patients with SARS-CoV-2 infections. ${ }^{4,12,13}$ Sacubitril in combination with valsartan was reported to increase NO bioavailability and reduce high sensitivity C-reactive protein, which can be additionally beneficial by improving microvascular function and reducing systemic inflammation.

In conclusion the findings from this study provides evidence for the potential novel role of neprilysin as a receptor for SARS-CoV-2 virus, which can be effectively targeted by currently approved neprilysin inhibitors.

\section{ACKNOWLEDGEMENT}

Research support from University College Dublin-Seed funding/ Output Based Research Support Scheme (R19862, 2019), Royal Society-UK (IES $\backslash R 2 \backslash 181067,2018)$ and Stemcology (STGY2708, 2020) is acknowledged.

\section{CONFLICT OF INTEREST}

The author declares that there is no conflict of interest.

\section{REFERENCES}

1. Esser N, Zraika S. Neprilysin inhibition: A new therapeutic option for type 2 diabetes?. Diabetologia. 2019;62(7):1113-22

2. Karoor V, Oka M, Walchak SJ, et al. Neprilysin regulates pulmonary artery smooth muscle cell phenotype through a platelet-derived growth factor receptor-dependent mechanism. Hypertension. 2013;61(4):921-30.

3. Nalivaeva NN, Zhuravin IA, Turner AJ. Neprilysin expression and functions in development, ageing and disease. Mech Ageing Dev. 2020;192:111363.

4. Acanfora D, Ciccone MM, Scicchitano $P$, et al. Neprilysin inhibitor-angiotensin II receptor blocker combination (sacubitril/valsartan): Rationale for adoption in SARS-CoV-2 patients. Eur Heart J Cardiovasc Pharmacother. 2020;6(3):135-6.

5. Mangiafico S, Costello-Boerrigter LC, Andersen IA, et al. Neutral endopeptidase inhibition and the natriuretic peptide system: An evolving strategy in cardiovascular therapeutics. Eur Heart J. 2013;34(12):886-93c.

6. Rice GI, Thomas DA, Grant PJ, et al. Evaluation of angiotensin-converting enzyme (ACE), its homologue ACE2 and neprilysin in angiotensin peptide metabolism. Biochem J. 2004;383(Pt 1):45-51.

7. Pavo N, Gugerell A, Goliasch G, et al. Increased granulocyte membrane neprily$\sin (C D 10)$ expression is associated with better prognosis in heart failure. Eur $J$ Heart Fail. 2019;21(4):537-9.

8. Shipp MA, Stefano GB, Switzer SN, et al. CD10 (CALLA)/neutral endopeptidase 24.11 modulates inflammatory peptide-induced changes in neutrophil morphology, migration, and adhesion proteins and is itself regulated by neutrophil activation. Blood. 1991;78(7):1834-41.

9. Borson DB, Brokaw JJ, Sekizawa K, et al. Neutral endopeptidase and neurogenic inflammation in rats with respiratory infections. J Appl Physiol 1989;66(6):2653-8.

10. Dempsey EC, Wick MJ, Karoor $\bigvee$, et al. Neprilysin null mice develop exaggerated pulmonary vascular remodeling in response to chronic hypoxia. Am J Pathol. 2009;174(3):782-96.

11. Song S, Zhang M, Yi Z, et al. The role of PDGF-B/TGF-beta1/neprilysin network in regulating endothelial-to-mesenchymal transition in pulmonary artery remodeling. Cell Signal. 2016;28(10):1489-501.

12. Tabaa EMM, Tabaa EMM. New putative insights into neprilysin (NEP)-dependent pharmacotherapeutic role of roflumilast in treating COVID-19. Eur J Pharmacol. 2020;889:173615.

13. Srivastava PK, Fonarow GC. In-Hospital Initiation of Angiotensin Receptor-Neprilysin Inhibitors-The Time Is Now. JAMA Cardiol. 2019;4(3):195-6.

14. Liczek M, Panek I, Damianski P, et al. Neprilysin inhibitors as a new approach in the treatment of right heart failure in the course of chronic obstructive pulmonary disease. Adv Respir Med. 2018;86(5):257-9.

15. Goothy SSK, Kumar AHS. Network Proteins of Angiotensin-converting Enzyme 2 but Not Angiotensin-converting Enzyme 2 itself are Host Cell Receptors for SARS-Coronavirus-2 Attachment. BEMS Reports. 2020;6(1):1-5.

16. Sharma A, Menche J, Huang CC et al. A disease module in the interactome explains disease heterogeneity, drug response and captures novel pathways and genes in asthma. Hum Mol Genet. 2015;24(11):3005-20.

17. Hruz T, Laule O, Szabo G, et al. Genevestigator v3: A reference expression database for the meta-analysis of transcriptomes. Adv Bioinformatics. 2008;420747.

18. Uhlen M, Fagerberg L, Hallstrom BM, et al. Proteomics: Tissue-based map of the human proteome. Science. 2015;347(6220):1260419. 
19. Kumar AHS. Molecular Docking of Natural Compounds from Tulsi (Ocimum sanctum) and neem (Azadirachta indica) against SARS-CoV-2 Protein Targets. BEMS Reports. 2020;6(1):11-3.

20. Yang Z, Lasker K, Schneidman-Duhovny D, et al. UCSF Chimera, MODELLER, and IMP: an integrated modeling system. J Struct Biol. 2012;179(3):269-78

21. Bordoli L, Kiefer F, Arnold K, et al. Protein structure homology modeling using SWISS-MODEL workspace. Nat Protoc. 2009;4(1):1-13.

22. Seeliger D, Groot DBL. Ligand docking and binding site analysis with PyMOL and AutodockNina. J Comput Aided Mol Des. 2010;24(5):417-22.

23. Sagar VK, Kumar AHS. Efficacy of Natural Compounds from Tinospora cordifolia against SARS-CoV-2 Protease, Surface Glycoprotein and RNA Polymerase. BEMS Reports. 2020;6(1):6-8.

24. Bharatham K, Bharatham N, Park KH, et al. Binding mode analyses and pharmacophore model development for sulfonamide chalcone derivatives, a new class of alpha-glucosidase inhibitors. J Mol Graph Model. 2008;26(8):1202-12.

25. Mishra A, Dey S. Molecular Docking Studies of a Cyclic Octapeptide-Cyclosaplin from Sandalwood. Biomolecules. 2019;9(11).

26. Stower H. Virological assessment of SARS-CoV-2. Nat Med. 2020;26(4):465.
27. Renu K, Prasanna PL, Gopalakrishnan VA. Coronaviruses pathogenesis, comorbidities and multi-organ damage: A review. Life Sci. 2020;255:117839.

28. Gregoriou GC, Patel SD, Winters BL, et al. Neprilysin Controls the Synaptic Activity of Neuropeptides in the Intercalated Cells of the Amygdala. Mol Pharmacol. 2020;98(4):454-61.

29. Kilroy D, Kumar AHS. Anatomical perspective on the loss of smell and taste sensation in SARS-CoV-2 infection. Anatomy. 2020;14(2):145-9.

30. Pavo N, Arfsten $\mathrm{H}$, Cho A, et al. The circulating form of neprilysin is not a gen eral biomarker for overall survival in treatment-naive cancer patients. Sci Rep. 2019;9(1):2554.

31. Burrell M, Henderson SJ, Ravnefjord A, et al. Neprilysin Inhibits Coagulation through Proteolytic Inactivation of Fibrinogen. PLoS One. 2016;11(7):e0158114.

32. Steiner MK. World Health Organization Class III COPD-associated pulmonary hypertension: Are we there yet in understanding the pathobiology of the disease?. Chest. 2009;136(3):658-9.

33. Wick MJ, Buesing EJ, Wehling CA, et al. Decreased neprilysin and pulmonary vascular remodeling in chronic obstructive pulmonary disease. Am J Respir Crit Care Med. 2011;183(3):330-40.

Cite this article : Kumar AHS. Molecular Profiling of Neprilysin Expression and its Interactions with SARS-CoV-2 Spike Proteins to Develop Evidence Base Pharmacological Approaches for Therapeutic Intervention. BEMS Reports. 2021;7(2):15-20. 